\title{
Comparative Leakage Analysis of GeOI FinFET and Ge Bulk FinFET
}

\author{
Vita Pi-Ho Hu, Member, IEEE, Ming-Long Fan, Student Member, IEEE, \\ Pin Su, Member, IEEE, and Ching-Te Chuang, Fellow, IEEE
}

\begin{abstract}
We present a comparative leakage analysis of germanium-on-insulator (GeOI) FinFET and germanium on bulk substrate FinFET (Ge bulk FinFET) at device and circuit levels. Band-to-band tunneling (BTBT) leakage-induced bipolar effect is found to result in an amplified BTBT leakage for GeOI FinFET. Device and circuit designs to mitigate the amplified BTBT leakage of GeOI FinFETs are suggested. The effectiveness of various high threshold voltage technology options including increasing channel doping, increasing gate length and drain-side underlap for leakage reduction is analyzed.
\end{abstract}

Index Terms-Band-to-band tunneling (BTBT) leakage, FinFET, germanium, germanium-on-insulator (GeOI).

\section{INTRODUCTION}

$\mathbf{F}$ inFET has emerged as the prime candidate for extremely scaled MOSFETs due to its superior control of shortchannel effects (SCEs) [1]-[3]. Germanium device offers high mobility [4]-[8]. However, due to its high permittivity and low bandgap, germanium device suffers from SCE and severe band-to-band tunneling (BTBT) leakage. Combining the advantages of the FinFET structure and the high mobility of Ge becomes a promising approach for future high-performance MOSFETs [9]-[12]. Si-on-insulator (SOI) FinFET and Si bulk FinFET have been studied [13]-[15], and the results showed that Si FinFET on bulk substrate with an optimized punch through stopper (PTS) doping [16] underneath the channel region can exhibit comparable performance and leakage with that on SOI substrate. However, a comparative leakage analysis between germanium-on-insulator (GeOI) FinFET and Ge bulk FinFET has rarely been seen.

In this work, the BTBT leakage current in the GeOI FinFET is shown to be amplified due to the bipolar effect, and various device design strategies for mitigating the bipolar gain $(\beta)$ and leakage current of GeOI FinFETs are examined. This work is organized as follows. Section II describes the device design, simulation methodology, and device characteristics. Section III investigates the device and circuit designs to suppress the

Manuscript received April 17, 2013; accepted August 7, 2013. Date of publication September 4, 2013; date of current version September 18, 2013. This work was supported in part by the National Science Council of Taiwan under Contract NSC 100-2628-E-009-024-MY2 and Contract NSC 102-2911I-009-302 (I-RiCE), and in part by the Ministry of Education in Taiwan under ATU Program. The review of this brief was arranged by Editor W. Tsai.

The authors are with the Department of Electronics Engineering and Institute of Electronics, National Chiao Tung University, Hsinchu 30010, Taiwan (e-mail: vitabee.ee93g@nctu.edu.tw; pinsu@faculty.nctu.edu.tw).

Color versions of one or more of the figures in this paper are available online at http://ieeexplore.ieee.org.

Digital Object Identifier 10.1109/TED.2013.2278032

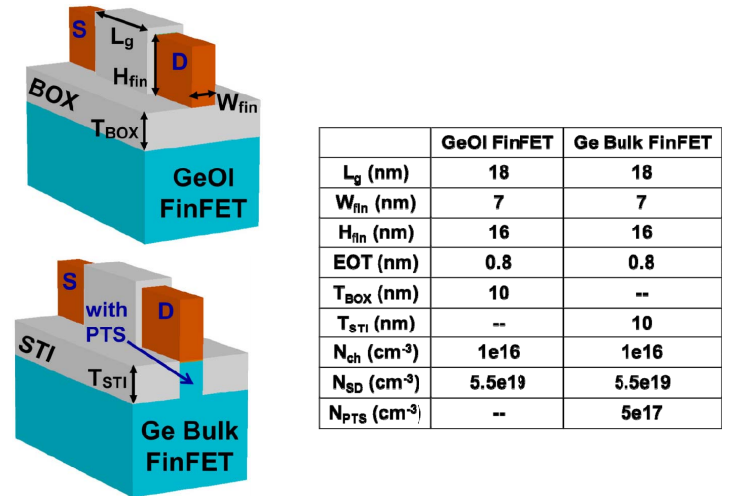

Fig. 1. Schematic view of GeOI and Ge bulk FinFETs, and device parameters used in this work. For Ge bulk FinFET, the PTS doping $\left(N_{\text {PTS }}\right)$ is adjusted to have comparable subthreshold swing compared with the GeOI FinFET.

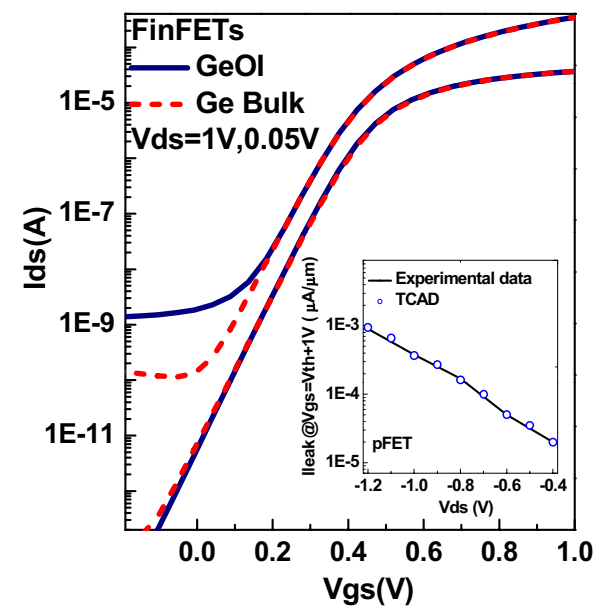

Fig. 2. $I_{\mathrm{ds}}$ versus $V_{\mathrm{gs}}$ characteristics of GeOI and Ge bulk FinFETs. Ge bulk FinFET uses PTS to control the subthreshold leakage. GeOI FinFET shows different $I_{\text {off }}$ compared with the Ge bulk FinFET at $V_{\mathrm{ds}}=1 \mathrm{~V}$. Inset: BTBT leakage current calibration at various $V_{\mathrm{ds}}[19]$.

amplified BTBT leakage due to the BTBT-induced bipolar effect. Section IV is the conclusion.

\section{Device Design And TCAD Simulation METHODOLOGY}

Fig. 1 shows the schematics of the GeOI and Ge bulk FinFETs and the device parameters used in this work. The PTS doping ( $\left.N_{\text {PTS }}\right)$ of the Ge bulk FinFET is adjusted to have comparable subthreshold swing compared with the GeOI FinFET, as shown in Fig. 2. 

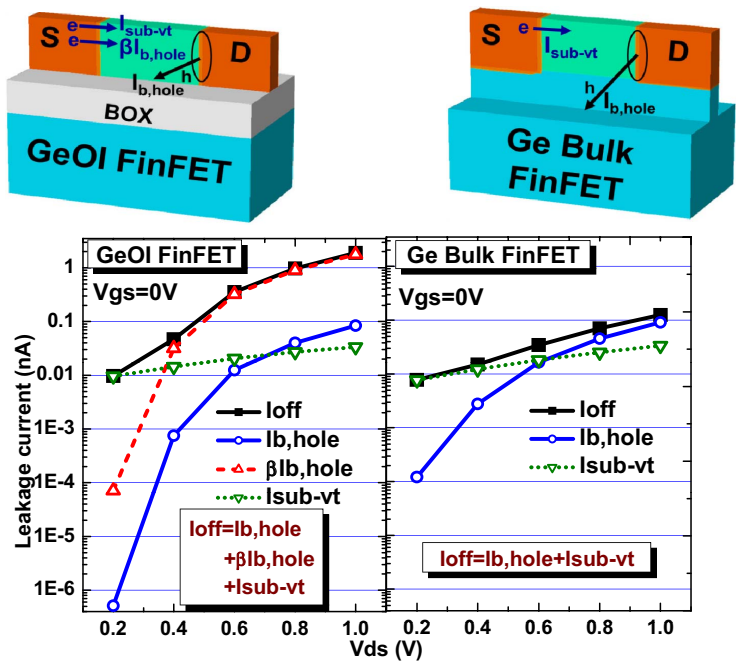

Fig. 3. Leakage components for GeOI FinFET and Ge bulk FinFET at various $V_{\mathrm{ds}}$. The BTBT induced bipolar leakage $\left(\beta \times I_{b}\right.$,hole $)$ is only observed in the GeOI FinFET.

The BTBT model [17], [18] is calibrated with the experimental data at various $V_{\mathrm{ds}}$ in [19], as shown in the inset of Fig. 2. The bandgap widening due to quantum confinement is considered for the 7-nm fin width $\left(W_{\text {fin }}\right)$ GeOI and $\mathrm{Ge}$ bulk FinFETs. The GeOI/Ge bulk FinFETs and circuits are analyzed using TCAD mixed-mode simulations [20].

Si bulk FinFET with optimized doping shows similar intrinsic device performance ( $I_{\text {off }}$ versus $I_{\text {on }}$ ) compared with the SOI FinFET at high and low $V_{\mathrm{ds}}$ [13], [14]. Fig. 2 shows that the GeOI FinFET exhibits comparable DIBL, subthreshold swing, and leakage at $V_{\mathrm{ds}}=0.05 \mathrm{~V}$ as compared with the Ge bulk FinFET with PTS. However, the leakage currents are different between the GeOI FinFET and the Ge bulk FinFET at $V_{\mathrm{ds}}=1 \mathrm{~V}$.

Fig. 3 shows the leakage components for the GeOI FinFET and Ge bulk FinFET at $V_{\mathrm{gs}}=0 \mathrm{~V}$ and $V_{\mathrm{ds}}=1 \mathrm{~V}$. The leakage components of the GeOI FinFETs include the BTBT

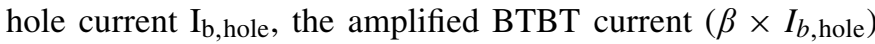
due to bipolar effect [21], and the subthreshold leakage current $\left(I_{\text {sub-vt }}\right)$, while the leakage components of Ge bulk FinFETs only include the BTBT hole current $\left(I_{b}\right.$,hole $)$, and the subthreshold leakage current $\left(I_{\text {sub-vt }}\right)$.

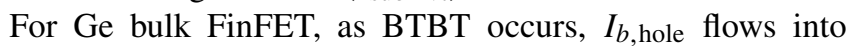
the substrate contact and hole concentration in the channel is much lower than the GeOI FinFET. Therefore, the BTBTinduced bipolar effect is not observed in the Ge bulk FinFETs. The total leakage $I_{\text {off }}$ for Ge bulk FinFET equals the sum of $I_{b \text {, hole }}$ and $I_{\text {sub-vt }}$. The leakage components of the GeOI FinFET and Ge bulk FinFET at various $V_{\mathrm{ds}}$ are shown in Fig. 3. The $I_{\text {off }}$ of GeOI FinFET is dominated by the BTBTinduced bipolar current $\left(\beta \times I_{b \text {,hole }}\right)$ at $V_{\mathrm{ds}}>0.4 \mathrm{~V}$. For $\mathrm{Ge}$ bulk FinFET, the $I_{\text {off }}$ is dominated by the BTBT leakage $I_{b}$, hole at $V_{\mathrm{ds}}>0.6 \mathrm{~V}$, and by the subthreshold leakage $\left(I_{\text {sub-vt }}\right)$ at $V_{\mathrm{ds}}<0.6 \mathrm{~V}$. Therefore, the BTBT-induced bipolar leakage needs to be considered for GeOI FinFET when it is compared with the Ge bulk FinFET.
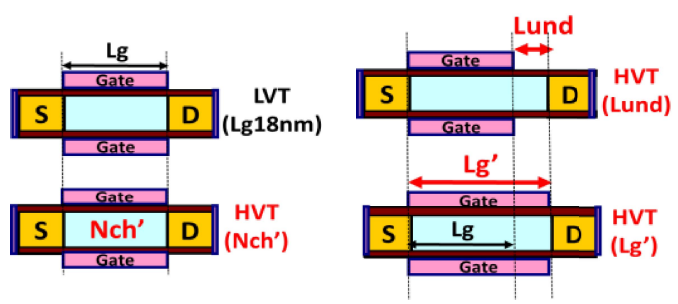

Fig. 4. Schematics of LVT, and HVT FinFETs options using drain-side underlap $\left(L_{\text {und }}=4 \mathrm{~nm}\right)$, increasing channel doping $\left(N_{\mathrm{ch}^{\prime}}=2 \mathrm{e} 18 \mathrm{~cm}^{-3}\right)$, and increasing gate length $\left(L_{g}^{\prime}=22 \mathrm{~nm}\right)$.

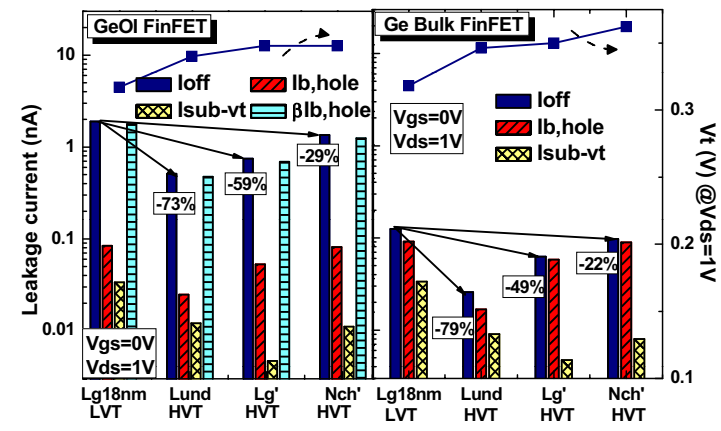

Fig. 5. Leakage comparisons of LVT and HVT FinFET options for GeOI and Ge bulk FinFETs at $V_{\mathrm{ds}}=1 \mathrm{~V}$. For GeOI and Ge bulk FinFETs at $V_{\mathrm{ds}}=1 \mathrm{~V}$, using HVT ( $\left.L_{\text {und }}\right)$ is the most effective way to reduce $I_{\text {off }}$.

\section{Mitigation of BTBT-Induced Bipolar LEAKAGE IN GeOI FinFETs}

Power-performance optimization often requires devices with multiple threshold voltages $\left(V_{\mathrm{t}}\right)$ [22]-[24]. High threshold voltage (HVT) transistors are typically employed for noncritical paths to reduce the leakage, while low threshold voltage (LVT) transistors are used for the critical paths. Several kinds of device design can be used to achieve HVT transistors. Asymmetric gate-to-source/drain underlap devices have been used in the static random access memory (SRAM) cell to adjust the threshold voltages and improve the cell leakage and stability [25], [26]. Increasing gate length was used in a microprocessor design [27]. Increasing the channel doping increases the total depletion charge in the device channel, hence increasing the $V_{t}$. Fig. 4 shows the schematics of (LVT, $L_{g}=18 \mathrm{~nm}$ ) and different HVT FinFETs options including drain-side underlap $\left(L_{\text {und }}=4 \mathrm{~nm}\right)$, increasing channel doping $\left(N_{\mathrm{ch}}^{\prime}=2 \mathrm{e} 18 \mathrm{~cm}^{-3}\right)$, and increasing gate length $\left(L_{g}^{\prime}=22 \mathrm{~nm}\right)$. The effectiveness of the HVT options to suppress the BTBT-induced bipolar leakage in the GeOI FinFET is examined.

Fig. 5 shows the leakage comparisons of LVT and HVT FinFET options for the GeOI and Ge bulk FinFETs at $V_{\mathrm{ds}}=1 \mathrm{~V}$. The leakage current of GeOI FinFET is dominated by the bipolar current $\left(\beta \times I_{b}\right.$,hole $)$, and the leakage current of Ge bulk FinFET is dominated by the BTBT current $I_{b \text {, hole }}$ at $V_{\mathrm{ds}}=1 \mathrm{~V}$. For GeOI and Ge bulk FinFETs at $V_{\mathrm{ds}}=1 \mathrm{~V}$, drain-side underlap [HVT $\left(L_{\text {und }}\right)$ ] shows significantly larger reduction $(73 \%$ and $79 \%)$ in $I_{\text {off }}$ compared with the increasing gate length $\left[\mathrm{HVT}\left(L_{g}^{\prime}\right)\right]$ and increasing channel doping [HVT $\left(N_{\text {ch }}^{\prime}\right)$ ], as shown in Fig. 5. This is because as drain-side 


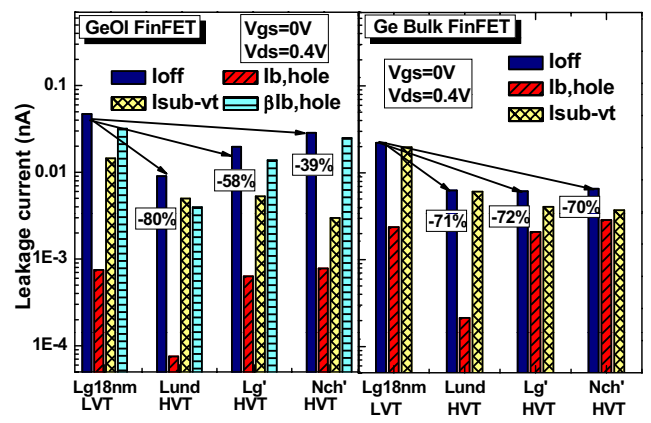

Fig. 6. Leakage comparisons of LVT and HVT FinFET options for GeOI and Ge bulk FinFETs at $V_{\mathrm{ds}}=0.4 \mathrm{~V}$. For GeOI FinFETs at $V_{\mathrm{ds}}=0.4 \mathrm{~V}$, using HVT ( $L_{\text {und }}$ ) is the most effective way to reduce $I_{\text {off }}$, while for Ge bulk FinFET at $V_{\mathrm{ds}}=0.4 \mathrm{~V}$, using $\operatorname{HVT}\left(L_{\mathrm{und}}\right)$, HVT $\left(L g^{\prime}\right)$, and $\operatorname{HVT}\left(N_{\mathrm{ch}}^{\prime}\right)$ shows comparable reduction in $I_{\text {off }}$.

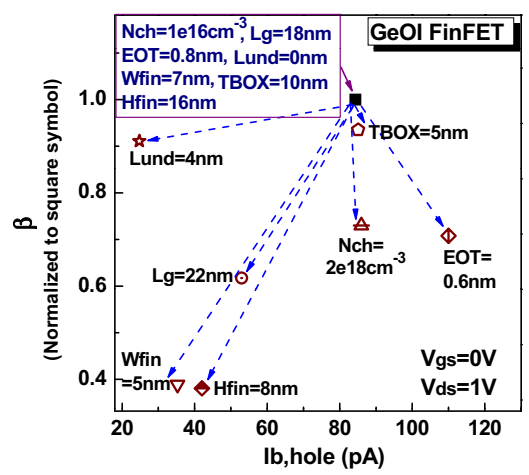

Fig. 7. Reducing $W_{\text {fin }}, H_{\text {fin }}$, and EOT, and increasing $N_{\text {ch }}$ and $L_{\mathrm{g}}$ can be used to reduce the bipolar gain of BTBT in GeOI FinFET.

underlap length $\left(L_{\text {und }}\right)$ increases, the BTBT width increases, thus reducing the $I_{b}$, hole and bipolar current $\left(\beta \times I_{b}\right.$, hole $)$ for the Ge bulk and GeOI FinFETs. Using HVT $\left(N_{\mathrm{ch}}^{\prime}\right)$ is the least effective way to reduce the $I_{\mathrm{off}}$ of the GeOI and Ge bulk FinFETs at $V_{\mathrm{ds}}=1 \mathrm{~V}$.

As $V_{\mathrm{ds}}$ scales down to $0.4 \mathrm{~V}$ (Fig. 6), the $I_{\text {off }}$ of LVT GeOI FinFET is dominated by the bipolar current $\left(\beta \times I_{b}\right.$, hole $)$, therefore, using HVT ( $L_{\text {und }}$ ) is the most effective to reduce $I_{\text {off }}$. On the other hand, for Ge bulk FinFET at $V_{\mathrm{ds}}=0.4 \mathrm{~V}$, using HVT $\left(L_{\text {und }}\right)$, HVT $\left(L_{g}^{\prime}\right)$, and $\operatorname{HVT}\left(N_{\text {ch }}^{\prime}\right)$ shows the comparable reduction in $I_{\text {off }}$ because the $I_{\text {off }}$ of $\mathrm{Ge}$ bulk FinFET is dominated by the subthreshold leakage $\left(I_{\text {sub-vt }}\right)$, and $I_{\text {sub-vt }}$ strongly depends on the threshold voltage. The effectiveness of using HVT $\left(L_{\text {und }}\right)$, HVT $\left(L_{g}^{\prime}\right)$, and HVT $\left(N_{\mathrm{ch}}^{\prime}\right)$ to reduce the leakage of the Ge bulk FinFET is different between $V_{\mathrm{ds}}=1 \mathrm{~V}$ and $V_{\mathrm{ds}}=0.4 \mathrm{~V}$ because the leakage of Ge bulk FinFET is dominated by the BTBT current at $V_{\mathrm{ds}}=1 \mathrm{~V}$ and by the subthreshold leakage current at $V_{\mathrm{ds}}=0.4 \mathrm{~V}$.

Fig. 7 shows the impact of different device design on the bipolar gain $(\beta)$ and the BTBT current $I_{b}$, hole of GeOI FinFET. The bipolar gain of the GeOI FinFET can be effectively suppressed by reducing the fin width $\left(W_{\text {fin }}\right)$, fin height $\left(H_{\text {fin }}\right)$ and EOT, and increasing channel doping $\left(N_{\mathrm{ch}}\right)$ and gate length $\left(L_{g}\right)$. Using drain-side underlap $\left(L_{\text {und }}\right)$ and reducing buried oxide thickness $\left(T_{\mathrm{BOX}}\right)$ show smaller impact in reducing the bipolar gain. This is because the body potential can be well controlled by gate as $W_{\text {fin }}, H_{\text {fin }}$ and EOT are reduced,

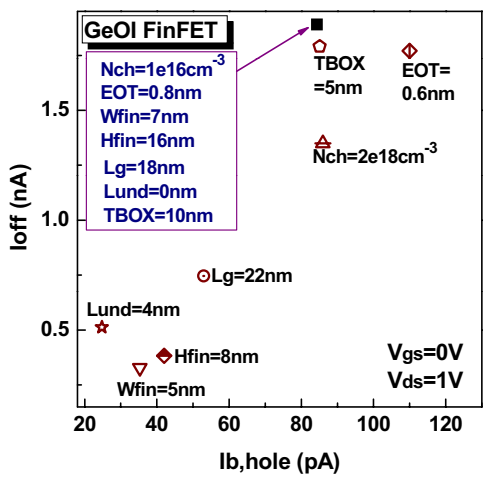

Fig. 8. Reducing $T_{\text {box }}$ and EOT shows less reduction in the $I_{\text {off }}$ of GeOI FinFETs.
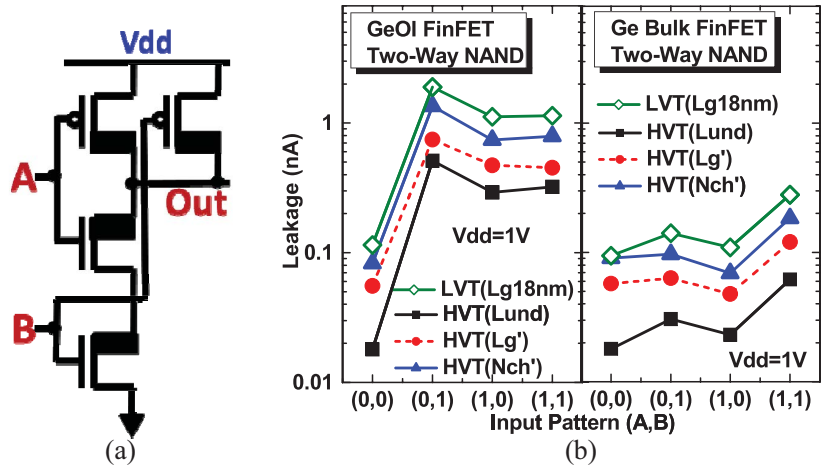

(b)

Fig. 9. (a) Schematic of HVT ( $L_{\text {und }}$ ) two-way NAND. The underlap region is marked with thick line. (b) Leakage comparisons of two-way NAND with $\mathrm{GeOI}$ and Ge bulk FinFETs for various input patterns at $V_{\mathrm{dd}}=1 \mathrm{~V}$.

thus reducing the bipolar gain. Although using $L_{\text {und }}$ shows small impact on reducing the bipolar gain, the $I_{\text {off }}$ of GeOI FinFET can be effectively reduced using $L_{\text {und }}$, because $L_{\text {und }}$ significantly reduce the BTBT leakage $I_{b}$, hole as shown in Fig. 8. Reducing $T_{\mathrm{BOX}}$ and EOT show less reduction in the $I_{\text {off }}$ of GeOI FinFETs since the BTBT leakage cannot be effectively suppressed by $T_{\mathrm{BOX}}$ and EOT reduction.

The HVT options shown in Fig. 4 are used to reduce the leakage of GeOI FinFET and Ge bulk FinFET two-way NAND circuits, as shown in Fig. 9. The schematic view of HVT ( $\left.L_{\text {nud }}\right)$ two-way NAND is shown in Fig. 9(a), where the underlap region is marked with black thick line. The underlap region is only placed at the drain junction, which has the significant band overlap and BTBT. Fig. 9(b) shows the GeOI FinFET and Ge bulk FinFET two-way NAND leakages with various input pattern at $V_{\mathrm{dd}}=1 \mathrm{~V}$. For input pattern $(\mathrm{A}, \mathrm{B})=(1,1)$, the leakage of GeOI FinFET two-way NAND is dominated by the BTBT-induced bipolar leakage $\left(\beta \times I_{b}\right.$,hole $)$ of two parallel off pFETs. For $(A, B)=(0,0)$, the leakage of two-way NAND is dominated by the two stacked off nFETs, and the leakage of the GeOI FinFET two-way NAND with $(\mathrm{A}, \mathrm{B})=(0,0)$ is reduced by one order of magnitude compared with GeOI FinFET two-way NAND with $(\mathrm{A}, \mathrm{B})=(1,1)$. Therefore, the amplified BTBT leakage of GeOI FinFET can be suppressed by transistor stacking [28]. For GeOI and Ge bulk FinFETs two-way NAND, using HVT ( $L_{\text {und }}$ ) shows lower leakage current than using $\operatorname{HVT}\left(L_{g}^{\prime}\right)$ and $\operatorname{HVT}\left(N_{\mathrm{ch}}^{\prime}\right)$. 


\section{CONCLUSION}

The BTBT leakage triggered parasitic lateral bipolar effect needs to be considered for the GeOI FinFET. Using drainside underlap is a more effective strategy to reduce leakage for $\mathrm{GeOI}$ and Ge bulk FinFETs compared with increasing gate length and channel doping. The bipolar gain of GeOI FinFETs can be suppressed by reducing the fin width, fin height and EOT, increasing channel doping and gate length, and by transistor stacking.

\section{REFERENCES}

[1] D. Hisamoto, W. Lee, J. Kedzierski, E. Anderson, H. Takeuchi, K. Asano, T. King, J. Bokor, and C. Hu, "A folded-channel MOSFET for deep-sub-tenth micron era," in IEDM Tech. Dig., Dec. 1998, pp. 1032-1034.

[2] (2012). International Technology Roadmap for Semiconductors (ITRS) [Online]. Available: http://www.itrs.net/

[3] E. Karl, Y. Wang, Y.-G. Ng, Z. Guo, F. Hamzaoglu, U. Bhattacharya, K. Zhang, K. Mistry, and M. Bohr, "A 4.6 GHz $162 \mathrm{Mb}$ SRAM design in $22 \mathrm{~nm}$ tri-gate CMOS technology with integrated active VMINenhancing assist circuitry," in Proc. ISSCC Dig. Tech., Feb. 2012, pp. $230-231$.

[4] D. Kuzum, T. Krishnamohan, A. Nainani, Y. Sun, P. A. Pianetta, H.-S. P. Wong, and K. C. Saraswat, "Experimental demonstration of high mobility Ge nMOS," in Proc. IEEE IEDM, Dec. 2009, pp. 453-456.

[5] T. Yamamoto, Y. Yamashita, M. Harada, N. Taoka, K. Ikeda, K. Suzuki, O. Kiso, N. Sugiyama, and S. Takagi, "High performance $60 \mathrm{~nm}$ gate length germanium p-MOSFETs with Ni germanide metal source/drain," in Proc. IEEE IEDM, Dec. 2007, pp. 1041-1043.

[6] P. Magnone, F. Crupi, M. Alioto, B. Kaczer, and B. De Jaeger, "Understanding the potential and the limits of germanium pMOSFETs for VLSI circuits from experimental measurements," IEEE Trans. Very Large Scale Integr. (VLSI) Syst., vol. 19, no. 9, pp. 1569-1582, Sep. 2011.

[7] P. Hashemi, W. Chern, H. S. Lee, J. T. Teherani, Y. Zhu, J. Gonsalvez, G. G. Shahidi, and J. L. Hoyt, "Ultrathin strained-Ge channel P-MOSFETs with high-k/metal gate and sub-1-nm equivalent oxide thickness," IEEE Electron Device Lett., vol. 33, no. 7, pp. 943-945, Jul. 2012 .

[8] G. Nicholas, T. J. Grasby, D. J. F. Fulgoni, C. S. Beer, J. Parsons, M. Meuris, and M. M. Heyns, "High mobility strained Ge pMOSFETs with high-k/metal gate," IEEE Electron Device Lett., vol. 28, no. 9, pp. 825-827, Sep. 2007.

[9] B. Liu, X. Gong, G. Han, P. S. Y. Lim, Y. Tong, Q. Zhou, U. Yang, N. Daval, C. Veytizou, D. Delprat, B.-Y. Nguyen, and Y.-C. Yeo, "Highperformance germanium $\Omega$-gate MuGFET with Schottky-barrier nickel germanide source/drain and low-temperature disilane-passivated gate stack," IEEE Electron Device Lett., vol. 33, no. 10, pp. 1336-1338, Oct. 2012.

[10] S.-H. Hsu, C.-L. Chu, W.-H. Tu, Y.-C. Fu, P.-J. Sung, H.-C. Chang, Y.-T. Chen, L.-Y. Cho, W. Hsu, G.-L. Luo, C. W. Liu, C. Hu, and F.L. Yang, "Nearly defect free Ge gate-all-around FETs on Si. substrates," in Proc. IEEE IEDM, Dec. 2011, pp. 825-828.

[11] M. J. H. Dal, G. Vellianitis, G. Doornbos, B. Duriez, T. M. Shen, C. C. Wu, R. Oxland, K. Bhuwalka, M. Holland, T. L. Lee, C. Wann, C. H. Hsieh, B. H. Lee, K. M. Yin, Z. Q. Wu, M. Passlack, and C. H. Diaz, "Demonstration of scaled Ge p-channel FinFETs integrated on Si," in Proc. IEEE IEDM, Dec. 2012, pp. 521-524.

[12] C.-T. Chung, C.-W. Chen, J.-C. Lin, C.-C. Wu, C.-H. Chien, and G.-L. Luo, "First experimental Ge CMOS FinFETs directly on SOI substrate," in Proc. IEEE IEDM, Dec. 2012, pp. 383-386.

[13] T. Chiarella, L. Witters, A. Mercha, C. Kerner, R. Dittrich, M. Rakowski, C. Ortolland, L. Ragnarsson, B. Parvais, A. Keersgieter, S. Kubicek, A. Redolfi, R. Rooyackers, C. Vrancken, and S. Brus, "Migrating from planar to FinFET for futher CMOS scaling: SOI or Bulk?" in Proc. ESSDERC, Sep. 2009, pp. 85-88.

[14] M. Poljak, V. Jovanovic, and T. Suligoj, "Improving bulk FinFET DC performance in comparison to SOI FinFET," Microelectron. Eng., vol. 86, no. 10, pp. 2078-2085, 2009.

[15] X. Sun, V. Moroz, N. Damrongplasit, C. Shin, and T.-J. K. Liu, "Variation study of the planar ground-plane bulk FMOSFET, SOI FinFET, and trigate bulk MOSFET designs," IEEE Trans. Electron Devices, vol. 58, no. 10 , pp. 3294-3299, Oct. 2011.
[16] K. Okano, T. Izumida, H. Kawasaki, A. Kaneko, A. Yagishita, T. Kanemura, M. Kondo, S. Ito, N. Aoki, K. Miyano, T. Ono, K. Yahashi, K. Iwade, T. Kubota, T. Matsushita, I. Mizushima, S. Inaba, K. Ishimaru, K. Suguro, K. Eguchi, Y. Tsunashima, and H. Ishiuchi, "Process integration technology and device characteristics of CMOS FinFET on bulk silicon substrate with sub-10 nm fin width and $20 \mathrm{~nm}$ gate length," in Proc. IEDM, Dec. 2005, pp. 721-724.

[17] G. Hellings, G. Eneman, R. Krom, B. Jaeger, J. Mitard, A. Keersgieter, T. Hoffmann, M. Meuris, and K. D. Meyer, "Electrical TCAD simulations of a germanium pMOSFET technology," IEEE Trans. Electron Devices, vol. 57, no. 10 , pp. 2539-2546, Oct. 2010

[18] V. P.-H. Hu, M.-L. Fan, P. Su, and C.-T. Chuang, "Comprehensive analysis of UTB GeOI logic circuits and 6T SRAM cells considering variability and temperature sensitivity," in Proc. IEEE IEDM, Dec. 2011, pp. 753-756.

[19] L. Hutin, C. Royer, J. Damlencourt, J. Hartmann, H. Grampeix, V. Mazzocchi, C. Tabone, B. Previtali, A. Pouydebasque, M. Vinet, and O. Faynot, "GeOI pMOSFETs scaled down to 30-nm gate length with record off-state current," IEEE Electron Device Lett., vol. 31, no. 3, pp. 234-236, Mar. 2010.

[20] Sentaurus TCAD, C2009-06 Manual, Synopsys, Mountain View, CA, USA, 2009.

[21] J. Chen, F. Assaderaghi, P. Ko, and C. Hu, "The enhancement of gateinduced-drain-leakage (GIDL) current in short-channel SOI MOSFET and its application in measuring lateral bipolar current gain" IEEE Electron Device Lett., vol. 13, no. 11, pp. 572-574, Nov. 1992.

[22] S. Mutoh, T. Douseki, Y. Matsuya, T. Aoki, S. Shigematsu, and J. Yamada, "1-V power supply high-speed digital circuit technology with multithreshold-voltage CMOS," IEEE J. Solid-State Circuits, vol. 30, no. 8, pp. 847-854, Aug. 1995.

[23] L. Wei, Z. Cen, and K. Roy, "Design and optimization of dual threshold circuits for low voltage, low power applications," IEEE Trans. Very Large Scale Integr. (VLSI) Syst., vol. 7, no. 1, pp. 16-24, Mar. 1999.

[24] K. Roy, S. Mukhopadhyay, and H. M. MeiMand, "Leakage current mechanisms and leakage reduction techniques in deep-submicrometer CMOS circuits," Proc. IEEE, vol. 91, no. 2, pp. 305-327, Feb. 2003.

[25] V. P.-H. Hu, M.-L. Fan, P. Su, and C.-T. Chuang, "Evaluation of static noise margin and performane of 6T FinFET SRAM cells with asymmetric gate to source/drain underlap devices," in Proc. IEEE Int. SOI Conf., Oct. 2010, pp. 1-2.

[26] S. Gupta, S. Choday, and K. Roy, "Exploration of device-circuit interactions in FinFET-based memories for sub-15 nm technologies using a mixed mode quantum simulation framework: Atoms to systems," in Proc. IEEE IEDM, Dec. 2011, pp. 757-760.

[27] S. Rusu, S. Tam, H. Muljono, D. Ayres, and J. Chang, "A dual-core multi-threded Xeon proessor with 16-MB L3 cache," in Proc. ISSCC Dig. Tech. Papers, Feb. 2005, pp. 315-324.

[28] V. P.-H. Hu, M.-L. Fan, P. Su, and C.-T. Chuang, "Band-to-band tunneling leakage suppression for ultra-thin-body GeOI MOSFETs using transistor stacking," IEEE Electron Device Lett., vol. 33, no. 2, pp. 197-199, Feb. 2012.

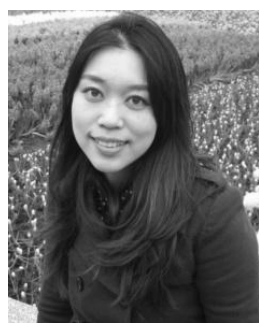

Vita Pi-Ho Hu (S'09-M'13) received the Ph.D. degree from the Department of Electronics Engineering \& Institute of Electronics, National Chiao Tung University, Hsinchu, Taiwan, in 2011.

She is currently an Assistant Researcher with National Chiao Tung University.

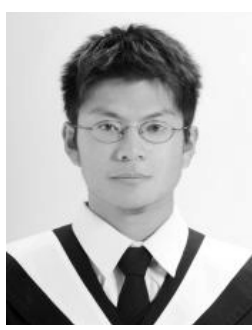

Ming-Long Fan (S'09) received the B.S. and M.S. degrees from the National Chiao Tung University, Hsinchu, Taiwan, respectively, where he is currently pursuing the Ph.D. degree in the Institute of Electronics. 


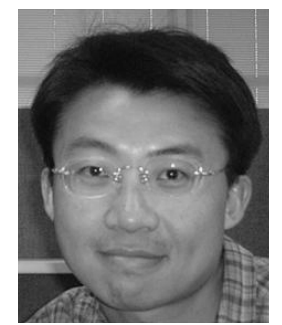

Pin Su (S'98-M'02) received the Ph.D. degree from the University of California, Berkeley, CA, USA.

$\mathrm{He}$ is currently a Professor with the Department of Electronics Engineering, National Chiao Tung University, Hsinchu, Taiwan.

Prof. Su serves in the technical committee of IEDM.

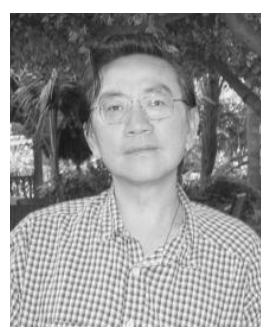

Ching-Te Chuang (S'78-M'82-SM'91-F'94) received the $\mathrm{Ph} . \mathrm{D}$. degree in electrical engineering from the University of California, Berkeley, CA, USA, in 1982.

$\mathrm{He}$ is currently a Chair Professor with the Department of Electronics Engineering, National Chiao Tung University, Hsinchu, Taiwan. 